\title{
A Feedback Based Closed Loop Circuit to Control Chaos in Nonlinear Circuits
}

\author{
Udayprakash Raghunath Singh ${ }^{1}$, Ghanshyam Purohit ${ }^{2}$ \\ ${ }^{1}$ Department of Electronics and Communication Engineering, Sir Padampat Singhania University, Bhatewar, Udaipur-313 601, India \\ ${ }^{2}$ Department of Physics, Sir Padampat Singhania University, Bhatewar, Udaipur-313 601, India
}

\begin{abstract}
This paper presents a simple circuit for control of chaos in nonlinear oscillator circuit. Numerical and circuit simulation results are used to visualize and illustrate the effectiveness of chaos control. Duffing Holmes oscillator circuit is considered as a nonlinear circuit.
\end{abstract}

Keywords: nonlinear dynamic, suppression of chaos, chaos control, Duffing Holmes oscillator, coupling.

\section{Introduction}

Over the past few decades, the complex and chaotic behavior of nonlinear dynamic system has attracted the attention of many researchers, scientists and engineers in both the academic area and industry. Most of the electronic/ electrical devices are nonlinear in nature that shows chaotic behavior. There are some practical situations where one needs to improve the performance of a dynamical system by avoiding / controlling chaos.

The deterministic chaos seems to explain many natural phenomena and there has been growing interest to use chaos profitably by synchronizing chaotic orbits [1] [2] due to its potential application in communication [3][4][5][6][7]. However, in many situations chaos is undesirable phenomenon, which may lead to irregular operation in physical systems. Thus from a practical point of view one would like to convert chaotic solutions into periodic or limit cycle solutions.

The strategies for the control of chaos can be classified into two main classes, which involve either stabilizing or suppressing chaos. The first category of methods includes the control of chaos by stabilizing the unstable periodic orbits embedded in a chaotic attractor such as OGY [8], proportional feedback [9], occasional proportional feedback [10] [11], continuous feedback [12] [13], delayed continuous feedback [14][15][16], extended delayed continuous feedback [17] and pulsed proportional feedback [18]. The second category of methods includes the control of chaos by converting chaotic behaviour into any one of the desired periodic behaviour exhibited by the system such as adaptive control algorithm [19] [20], parametric perturbation [21], using second periodic force [22], weak feedback control [23], addition of noise [24] and constant feedback [25], etc. The algorithms used for control of chaos have also been classified into closed loop or feedback methods [26] [9] and open loop or non-feedback methods [21]. In a closed loop method the perturbation is based upon the prior knowledge of the state of the system whereas in a open loop method the perturbation is independent of the knowledge of the state of the system.
Patidar et al [27] presented a simple method to control chaotic oscillations of systems described by second or third order nonlinear differential equations. Their method was a closed loop or feedback method and can be classified as a suppressing chaos algorithm, as it converts chaotic behavior into a desired periodic behavior. They do not require any prior knowledge of the system dynamics, such as location of unstable periodic orbits, periodic orbits or stable fixed points. They only required a weak periodic oscillator of the same kind as the chaotic oscillator. They introduced a mutual coupling between the state variables of both chaotic and weak periodic oscillators. They have introduced a new parameter called coupling strength to control the mutual coupling between the chaotic and weak periodic oscillators.

Based on the literature review it is observed that the feedback control method is one of the most effective methods of chaos control. The algorithm given by [27] is one of the effective methods for the chaos control. The work presented in this paper proposes a new circuit based on the algorithm introduced by [27] for control of chaos in Duffing Holmes oscillator circuit. The effectiveness of the proposed work is illustrated through matlab simulation and PSpice circuit simulation.

\section{Duffing Holmes Oscillator Circuit [28]}

The Duffing-Holmes oscillator is given either by the second order nonautonomous differential equation [26][29][30][31]:

$$
\ddot{x}+b \dot{x}-x+x^{3}=a \sin \omega t
$$

or by an equivalent set of two first order nonautonomous equations

$$
\begin{gathered}
\dot{x}=y \\
\dot{y}=F(x)-b y+a \sin \omega t
\end{gathered}
$$

with $F(x)=x-x^{3}$. In (1) and (2) b, a, and $\omega$ are the damping coefficient, the amplitude and the frequency of the external driving force, respectively. 


\section{International Journal of Science and Research (IJSR) \\ ISSN (Online): 2319-7064}

Index Copernicus Value (2013): 6.14 | Impact Factor (2014): 5.611

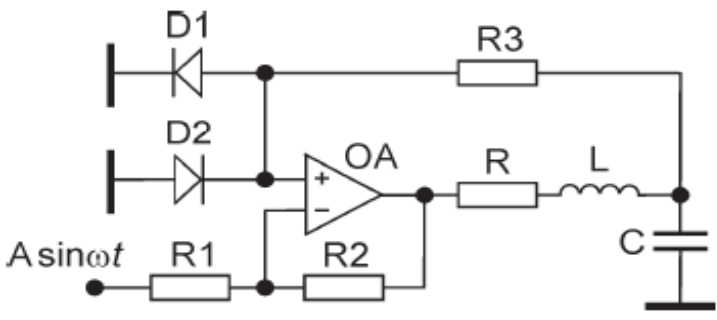

Figure 1: Duffing Holmes Oscillator Circuit

Differential equations describing the circuit can be easily obtained using the Kirchhoff's

laws: -

$$
\begin{gathered}
c \frac{d V c}{d t}=I_{L}, \\
L \frac{d I_{L}}{d t}=F_{E}(V C)-I_{L} R+A \sin (\omega t-\pi)
\end{gathered}
$$

where, $V C$ is the voltage across the capacitor $\mathrm{C}$ and $I_{L}$ is the current through the inductor $\mathrm{L}$.

$$
\begin{gathered}
F_{E}(V c)=-\left(\mathrm{Vc}+\mathrm{kV}^{*}\right), \text { for } \mathrm{Vc}<-\mathrm{V} \\
=(\mathrm{k}-1) \mathrm{Vc}, \text { for }-\mathrm{V} \leq \mathrm{Vc} \leq \mathrm{V}^{*} \\
=-\left(\mathrm{Vc}^{*}-\mathrm{kV}^{*}\right), \text { for } \mathrm{Vc}>\mathrm{V}^{*}
\end{gathered}
$$

where $k=(R 2 / R 1)+1$ is the gain of the amplifying stage and $\mathrm{V}^{*}$ is the voltage drop across an opened diode (for silicon diode $\mathrm{V}^{*}=0.5 \mathrm{~V}$ at $0.1 \mathrm{~mA}$ ).

\section{Chaos Control}

The schematic representation of an algorithm used for suppression of chaos is shown in Fig. 2. It is a two oscillator system in which one is a chaotic which is to be suppressed and another one is a weak periodic but of the same kind of the chaotic oscillator. Here the mixing of signals is controlled by coupling strength parameter (c). In this algorithm the mixing of signals is bidirectional i.e. the percentage of mixing depends upon the value of mutual coupling strength (i.e. if c $=0.5$ then the percentage of mixing will be only $50 \%)$.

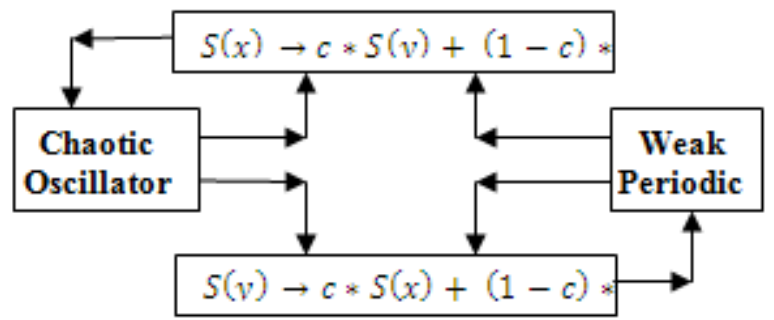

Figure 2: Schematic representation of the algorithm for suppression of chaos.

We have constructed an analog circuit equivalent to the schematic representation of the algorithm for suppression of chaos given in Fig. 2 with Duffing Holmes Oscillators as chaotic oscillator and periodic oscillator by using conventional operational amplifiers and diodes, which is shown in Fig. 3.

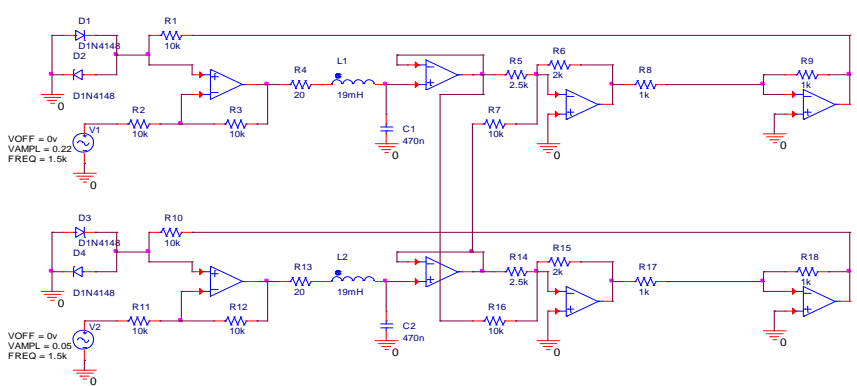

Figure 3: Circuit Diagram for the control of chaos in Duffing-Holmes oscillator

The circuit is constructed using conventional operational amplifiers and diodes. The operational amplifiers used are op-amp $\mu \mathrm{A} 741 \mathrm{C}$ and diodes used are D1N4148. The values of various resistors, inductors and capacitors used in the circuit are as given below: -

$$
\begin{aligned}
& R 1=R 2=R 3=R 7=R 10=R 11=R 12= \\
& R 16=10 k \Omega, R 4=R 13=20 \Omega, R 8=R 9= \\
& R 17=R 18=1 k \Omega, \text { Variable resistors: } R 5= \\
& R 6=R 14=R 15=10 k \Omega, L 1=L 2= \\
& 19 \mathrm{mH} \text { and } \mathrm{C} 1=\mathrm{C} 2=470 \mathrm{nF} . \text { Here } \mathrm{c}=\frac{\mathrm{R} 6}{\mathrm{R} 7}= \\
& \frac{\mathrm{R} 15}{\mathrm{R} 16} \text { and }(1-\mathrm{c})=\frac{\mathrm{R} 6}{\mathrm{R} 5}=\frac{\mathrm{R} 15}{\mathrm{R} 14}
\end{aligned}
$$

\section{Simulation Results}

We present the results of PSpice simulation study and their comparison with the numerical results in Fig. 4 - Fig. 8 . When $\mathrm{c}=0$, the circuit of Fig. 3 behaves as normal Duffing Holmes Oscillators. In these figures we can see that for different forcing amplitudes it generates chaotic signals. These chaotic signals are suppressed by mixing (as per the algorithm) a very small periodic signal generated by another Duffing Holmes Oscillator with small forcing amplitude of $0.005 \mathrm{~V}$. The numerical results are obtained by using the damping coefficient and the frequency of the external driving force as $b=0.1$ and $\omega=1.3$ respectively for different values of forcing amplitude $\mathrm{F}$, at which chaotic signals are generated.

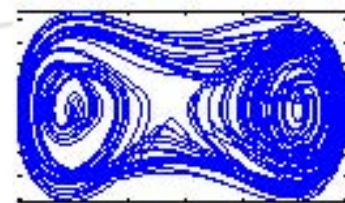

(a) $\mathrm{F}=0.2, \mathrm{c}=0$

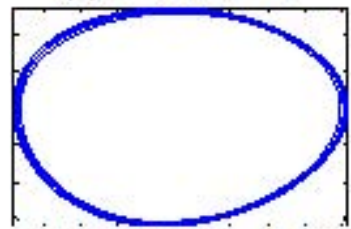

(c) $\mathrm{F}=0.2, \mathrm{c}=0.2$

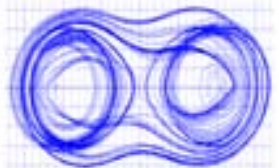

(b) $\mathrm{F}=0.2, \mathrm{c}=0$

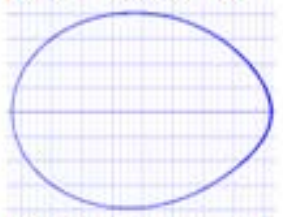

(d) $\mathrm{F}=0.2, \mathrm{c}=0.2$
Figure 4: Phase Plots ( $\dot{x}$ vs $x$ i.e. $\dot{x}$ on $\mathrm{Y}$-axis and $x$ on $\mathrm{X}$ axis): (a) and (c) are Numerical Simulation Result and (b) and (d) are PSpice Simulation Result.

We can see in Fig. 4, the chaotic signal generated at forcing amplitude $F=0.2$ ((a) and (b)) is suppressed by mixing of

\section{Volume 5 Issue 3, March 2016}




\section{International Journal of Science and Research (IJSR) \\ ISSN (Online): 2319-7064 \\ Index Copernicus Value (2013): 6.14 | Impact Factor (2014): 5.611}

signals with $20 \%$ coupling strength parameter (c) as shown in figures (c) and (d).

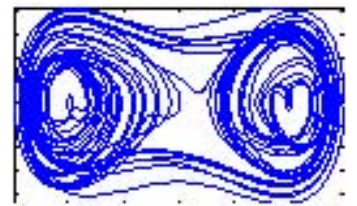

(a) $\mathrm{F}=0.22, \mathrm{c}=0$

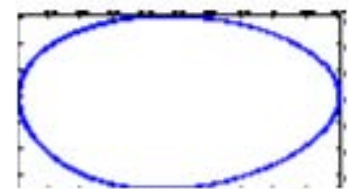

(c) $\mathrm{F}=0.22, \mathrm{c}=0.25$

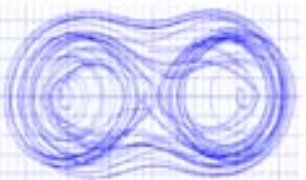

(b) $\mathrm{F}=0.22, \mathrm{c}=0$

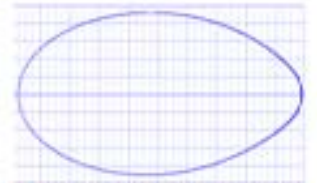

(d) $\mathrm{F}=0.22, \mathrm{c}=0.25$
Figure 5: Phase Plots ( $\dot{x}$ vs $x$ i.e. $\dot{x}$ on $\mathrm{Y}$-axis and $x$ on $\mathrm{X}$ axis): (a) and (c) are Numerical Simulation Result and (b) and (d) are PSpice Simulation Result.

We can see in Fig. 5, the chaotic signal generated at forcing amplitude $\mathrm{F}=0.22$ ((a) and (b)) is suppressed by mixing of signals with $25 \%$ coupling strength parameter (c) as shown in figures (c) and (d).

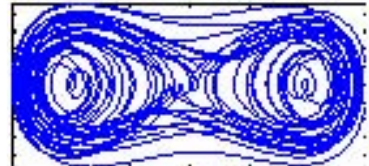

(a) $\mathrm{F}=0.24, \mathrm{c}=0$

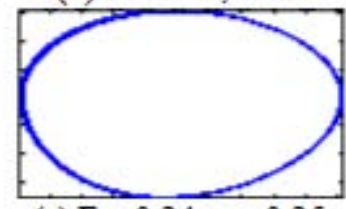

(c) $\mathrm{F}=0.24, \mathrm{c}=0.25$

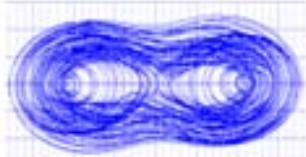

(b) $\mathrm{F}=0.24, \mathrm{c}=0$

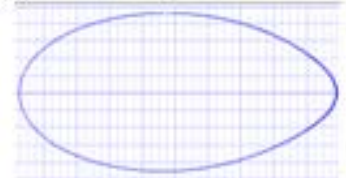

(d) $F=0.24, c=0.25$
Figure 6: Phase Plots ( $\dot{x}$ vs $x$ i.e. $\dot{x}$ on $\mathrm{Y}$-axis and $x$ on $\mathrm{X}$ axis): (a) and (c) are Numerical Simulation Result and (b) and (d) are PSpice Simulation Result.

We can see in Fig. 6, the chaotic signal generated at forcing amplitude $\mathrm{F}=0.24$ ((a) and (b)) is suppressed by mixing of signals with $25 \%$ coupling strength parameter (c) as shown in figures (c) and (d).

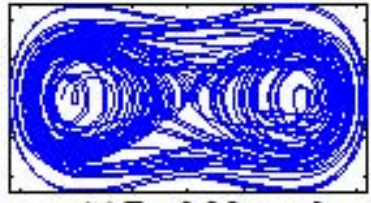

(a) $\mathrm{F}=0.35, \mathrm{c}=0$

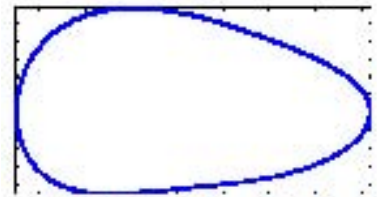

(c) $\mathrm{F}=0.35, \mathrm{c}=0.38$

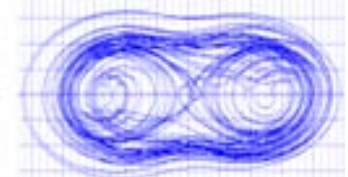

(b) $\mathrm{F}=0.35, \mathrm{c}=0$

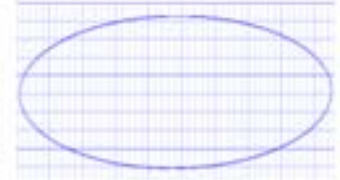

(d) $\mathrm{F}=0.35, \mathrm{c}=0.38$
Figure 7: Phase Plots ( $\dot{x}$ vs $x$ i.e. $\dot{x}$ on $\mathrm{Y}$-axis and $x$ on $\mathrm{X}$ axis): (a) and (c) are Numerical Simulation Result and (b) and (d) are PSpice Simulation Result.
We can see in Fig. 7, the chaotic signal generated at forcing amplitude $\mathrm{F}=0.35$ ((a) and (b)) is suppressed by mixing of signals with $38 \%$ coupling strength parameter (c) as shown in figures (c) and (d).
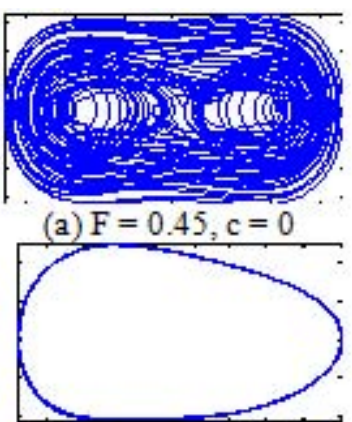

(c) $\mathrm{F}=0.45, \mathrm{c}=0.40$

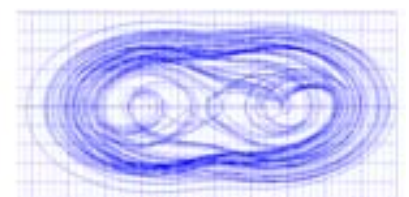

(b) $\mathrm{F}=0.45, \mathrm{c}=0$

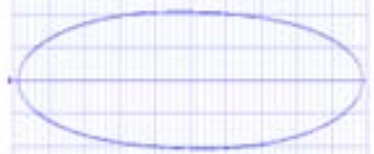

(d) $\mathrm{F}=0.45, \mathrm{c}=0.40$
Figure 8: Phase Plots ( $\dot{x}$ vs $x$ i.e. $\dot{x}$ on $\mathrm{Y}$-axis and $x$ on $\mathrm{X}$ axis): (a) and (c) are Numerical Simulation Result and (b) and (d) are PSpice Simulation Result.

We can see in Fig. 8, the chaotic signal generated at forcing amplitude $\mathrm{F}=0.45$ ((a) and (b)) is suppressed by mixing of signals with $40 \%$ coupling strength parameter (c) as shown in figures (c) and (d).

\section{Conclusion}

In this paper we propose a new circuit to control chaos in nonlinear circuits. Using Duffing Holmes Oscillator as nonlinear circuit, we have demonstrated in numerical and PSpice simulations that chaos can be controlled in nonlinear circuit. We have observed that by varying mutual coupling strength we can control chaos obtained at various external forcing amplitude.

\section{References}

[1] Pecora, L.M., Carroll, T.L. [1991] "Driving systems With Chaotic Signals", Phys. Rev. A 44, 2374.

[2] Pecora, L.M., Carroll, T.L., Johnson, G.A., Mar, D.J., Heagy, J.F. [1997] "Fundamentals of synchronization in chaotic systems, concepts and applications", Chaos 7, 520.

[3] Parlitz, U., Chua, L.O., Kocarev, L. [1992] "Transmission of digital signals by chaoyic synchronization", Int. J. Bifur. Chaos 2, 973.

[4] Cuomo, K., Oppenheim, A.V. [1993] "Circuit implementation of synchronized chaos with applications to communications",Phys. Rev. Lett. 71, 65.

[5] Murali,K., Laksmanan, M. [1993] "Transmission of signals by synchronization in a chaotic Van der PolDuffing oscillator", Phys. Rev. E 48, R1624.

[6] Kocarev, L., Parlitz, U. [1995] "General approach for chaotic synchronization with applications to communication", Phys. Rev. Lett. 74,5028.

[7] Perez, G., Cerderia, H.A. [1995] "Extracting messages masked by chaos", Phys. Rev. Lett. 74, 1970.

[8] Ott, E., Grebogi, C., Yorke, J.A. [1990] "Controlling chaos", Phys. Rev. Lett. 64,1196. 


\section{International Journal of Science and Research (IJSR) \\ ISSN (Online): 2319-7064}

Index Copernicus Value (2013): 6.14 | Impact Factor (2014): 5.611

[9] Petrov,V., Gaspar, Y., Masere, J., Showalter, K. [1993] "Controlling chaos in the Belousov-Zhabotinsky reaction”, Nature 361, 240.

[10] Hunt, E.R. [1991] "Stabilizing high-period orbits in a chaotic system: The diode resonator", Phys. Rev. Lett. 67, 1953.

[11] Roy, R., Murphy, T.W., Maier, T.D., Gills, Z., Hunt, E.R. [1992] "Dynamical control of a chaotic laser: experimental stabilization of a globally coupled system", Phys. Rev. Lett. 68, 1259.

[12] Pyragas, K. [1992] "Continuous control of chaos by selfcontrolling feedback", Phys. Lett. A 170, 421

[13] Qu, Z., Hu, G., Ma, B. [1993] "Note on continuous chaos control", Phys. Lett. A 178,265.

[14]Bielawaski, S., Dreoizier, D., Glorieux, P. [1994] "Controlling unstable periodic orbits by a delayed continuous feedback", Phys. Rev. E 49, R971.

[15] Pyragas, K., Tamasevicius, A. [1993] "Experimental control of chaos by self-controlling feedback", Phys. Lett. A 180, 99.

[16] Just, W., Mockel, J., Reckwerth, D., Reiboldnand, E., Benner, H. [1998] "Delayed feedback control of periodic orbits in autonomous systems", Phys. Rev. Lett. 81, 562.

[17] Pyragas, K. [1995] "Control of chaos via extended delay feedback", Phys. Lett. A 206, 323.

[18] Gumez, J., Matias, M. A. [1993] "Control of chaos in unidimensional maps", Phys. Lett. A 181, 29.

[19] Huberman, B.A., Lumer, L. [1990] "Dynamics of adaptive systems", IEEE Trans. Circ. Syst. 37, 547.

[20] Sinha, S., Ramaswamy, R., Subba Rao, J. [1990] "Adaptive control in nonlinear dynamics", Physica D 43, 118.

[21] Lima, R., Pettini, M. [1990] "Suppression of chaos by resonant parametric perturbations", Phys. Rev. A 41, 726.

[22] Pehlivan, Ihsan, Wei, Zhouchao [2012] "Analysis, nonlinear control, and chaos generator circuit of another strange chaotic system", Turk J. Elec Eng \& Comp Sci, 20 (2).

[23] Braiman, Y., Goldhirsch, I. [1991] "Taming chaotic dynamics with weak periodic perturbation", Phys. Rev. Lett. 66, 2545.

[24] Singer, J., Wang, Y.Z., Bau, H.H. [1991] "Controlling a chaotic system”, Phys. Rev. Lett. 66, 1123.

[25] Rajasekar, S., Lakshmanan, M. [1993] "Algorithms for controlling chaotic motion: application for the BVP oscillator", Physica D 67, 282;

[26] Parthasarthy, S., Sinha, S. [1995] "Controlling chaos in unidimensional maps using constant feedback", Phys. Rev. E 51, 6239.

[27] Ott E. [1993] "Chaos in Dynamical Systems”, Cambridge University Press, Cambridge.

[28] Patidar, V., Pareek, N. K., Sud, K. K. [2002] "Suppression of chaos using mutual coupling", Phys. Lett. A 304, 121- 129.

[29] Tamaseviciute, E., Tamasevicius, A., Mykolaitis, G., Bumeliene, S., Lindberg, E. [2008] "Analogue Electrical Circuit for Simulation of the Duffing-Holmes equation", Nonlinear Analysis: Modelling and Control, 13 (2), 241252.
[30] Holmes, P. [1979] "A nonlinear oscillator with a strange attractor", Phil. Trans. Roy. Soc. London A, 292, 419448.

[31]Moon, F. C. [1987] "Chaotic Vibrations: An Introduction for Applied Scientists and Engineers", Wiley,New York.

[32] Alligood, K. T., Sauer, T.D., Yorke, J.A. [2000] “Chaos - an Introduction to Dynamical Systems", Springer, New York, Berlin.

\section{Author Profile}

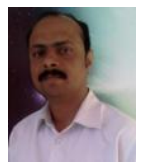

Udayprakash Raghunath Singh is working as an Associate Professor of Electronics \& Communication Engineering and Controller of Examination at Sir Padampat Singhania University, Udaipur, India. $\mathrm{He}$ has obtained B.E. (Industrial Electronics) from Nagpur University, Nagpur, India in 2000 and M.Tech (VLSI Design) from MITS Lakshmangarh, Sikar, Rajasthan, India in 2009. He has about 15 years of teaching \& research experience. He is working in the field of Nonlinear Dynamics and Circuits.

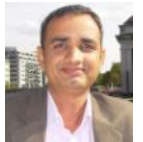

Ghanshyam Purohit is working as Professor of Physics and Associate Dean - Research at Sir Padampat Singhania University, Udaipur, India. He has obtained $\mathrm{Ph}$. D. degree in theoretical atomic physics from M. L. S. University, Udaipur in 2005. He has over 40 research publications in the field of atomic physics, non-linear dynamics and chaos. Dr. Purohit has visited ICTP, Trieste (Italy), Max Planck Institute for Nuclear Sciences, Heidelberg (Germany), ITAMP, Harvard University, Cambridge ( USA) as visiting scientist, besides this he has presented research work at various international conferences held in UK, USA, Japan, Germany, France, Austria, Italy, Sweden, Switzerland, Ireland, China etc. 